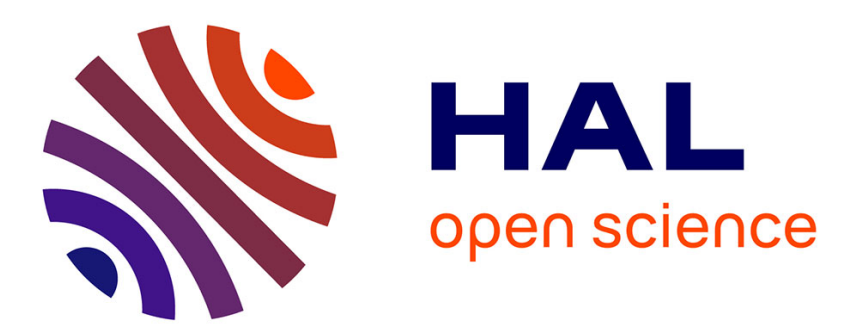

\title{
Terahertz Radiation from a Longitudinal Electric Field Biased Femtosecond Filament in Air
}

\author{
Yi Liu, Shaojie Liu, Aurélien Houard, André Mysyrowicz, Vladimir T \\ Tikhonchuk
}

\section{- To cite this version:}

Yi Liu, Shaojie Liu, Aurélien Houard, André Mysyrowicz, Vladimir T Tikhonchuk. Terahertz Radiation from a Longitudinal Electric Field Biased Femtosecond Filament in Air. Chinese Physics Letters, 2020, 37, 10.1088/0256-307x/37/6/065201 . hal-02993245

HAL Id: hal-02993245

https: / hal-ensta-paris.archives-ouvertes.fr/hal-02993245

Submitted on 16 Dec 2020

HAL is a multi-disciplinary open access archive for the deposit and dissemination of scientific research documents, whether they are published or not. The documents may come from teaching and research institutions in France or abroad, or from public or private research centers.
L'archive ouverte pluridisciplinaire HAL, est destinée au dépôt et à la diffusion de documents scientifiques de niveau recherche, publiés ou non, émanant des établissements d'enseignement et de recherche français ou étrangers, des laboratoires publics ou privés. 


\title{
Terahertz radiation from a longitudinal electric field biased femtosecond filament in air
}

\author{
Yi Liu ${ }^{1,2,}$, Shaojie Liu ${ }^{1}$, Aurélien Houard ${ }^{2}$, André Mysyrowicz², Vladimir T. Tikhonchuk ${ }^{3,4}$ \\ ${ }^{1}$ Shanghai Key Lab of Modern Optical System, University of Shanghai for Science and Technology, \\ 516, Jungong Road, 200093 Shanghai, China \\ ${ }^{2}$ Laboratoire d'Optique Appliquée, ENSTA, Ecole Polytechnique, CNRS, Institut Polytechnique de Paris, \\ 828 Boulevard des Maréchaux, 91762 Palaiseau cedex, France \\ ${ }^{3}$ Centre Lasers Intenses et Applications, University of Bordeaux-CNRS-CEA, 351 Cours de la Liberation, \\ 33405 Talence cedex, France \\ ${ }^{4}$ ELI-Beamlines, Institute of Physics, Czech Academy of Sciences, 25241 Dolni Břežany, Czech Republic \\ *Corresponding author:*yi.liu@usst.edu.cn
}

\begin{abstract}
The Terahertz (THz) temporal waveform and spectrum from a longitudinal electrically biased femtosecond filament is studied experimentally. The initial direction of the electron motion inside the unbiased filament plasma is deduced from the transformation of the $\mathrm{THz}$ temporal waveform with applied fields of opposite polarities. Furthermore, a spectrum shift to lower frequency of the THz spectrum is observed in the presence of a biased field. It agrees well with theoretical predictions.
\end{abstract}

Terahertz radiation from plasma in air induced by laser pulses was first demonstrated by Hamster and co-workers about 25 years ago [1]. In recent years, the THz field from a long plasma channel, a filament, generated by an intense femtosecond laser has attracted much attention [2-11]. This air plasma $\mathrm{THz}$ source holds several unique advantages comparing to the traditional technique including photoconductive antenna and optical rectification with optical-electrical crystal. With femtosecond filament, the $\mathrm{THz}$ source can be positioned far away from the laser system, bypassing the detrimental absorption of the $\mathrm{THz}$ field by the water vapor during propagation in ambient air $[12,13]$. At the same time, high power laser pulses up to several Terawatts can be employed for $\mathrm{THz}$ generation without damage of emitter, which is not possible with the traditional techniques [12]. However, the conversion efficiency of this single-color filamentation method has been measured to be low, on the order of $10^{-9}[14,15]$. Several methods have been demonstrated to enhance the $\mathrm{THz}$ field, such as application of transverse or longitudinal electric field [4, 16-17], bifilamentation technique [18], and two-colors or multiple color excitation [2, 3, 5-11, 19- 
21]. In particular, the presence of a longitudinal field can enhance the intensity of the low frequency components by 1,000 times [17]. Furthermore, the physical properties of the $\mathrm{THz}$ emission from filaments remain the same as that excited with a single-color femtosecond pulse, including the characteristic radial polarization and hollow cone radiation pattern. It has been attributed to an enhanced transition-Cherenkov (TC) radiation from the filament plasma [17].

In this letter, we report the characterization of the temporal waveform and spectrum of the $\mathrm{THz}$ radiation from longitudinally electrical biased filaments. In the previous study, the characterization was performed with a heterodyne detector working at $0.1 \mathrm{THz}$ with a bandwidth of $4 \mathrm{GHz}$, limiting the insight into the enhanced $\mathrm{THz}$ radiation. Here, the full temporal form of the $\mathrm{THz}$ emission is recorded by electro-optical detection. It is revealed that in the presence of electric fields with opposite polarities, the time domain $\mathrm{THz}$ waveform experiences a $180^{\circ}$ phase shift. From these measurements, the initial direction of the electron motion inside a free filament is inferred to be toward the laser. Furthermore, the THz spectra from filaments with an external field show a spectrum shift towards lower frequency. Both effects are well explained by the theoretical Cerenkov-transition model.

In the experiment, a Chirped Pulse Amplification (CPA) femtosecond laser system was employed. This femtosecond laser system delivers $35 \mathrm{fs}$ pulses at $800 \mathrm{~nm}$, with pulse energy up to $8 \mathrm{~mJ}$. The pulses are split in two beams with a 90/10 beam splitter. In our experiment, up to $3 \mathrm{~mJ}$ pulses was focused by a $180 \mathrm{~mm}$ convex lens to generate a plasma filament in ambient air. The length of the plasma filament varied from 3 to $8 \mathrm{~mm}$, depending on the incident power. Two circular copper electrodes of $40 \mathrm{~mm}$ diameter with central holes were mounted on the filament, as presented in Fig. 1. The two electrodes were separated by about $15 \mathrm{~mm}$, with the filament placed around the middle. The diameters of the holes on the two electrodes were 3 and $10 \mathrm{~mm}$, respectively. A larger opening at the exit ensured that the THz field was not blocked by the electrode plate (see Fig. 1 of Ref. 12). A static voltage was applied to the exit electrode and the incident electrode was grounded. A high resistance silicon plate was employed to block the fundamental pump pulse after the plasma, while leaving the $\mathrm{THz}$ emission pass through. The $\mathrm{THz}$ emission 
from the biased filaments was first collimated by an off-axis parabolic mirror with focal length of $120 \mathrm{~mm}$. It was then focused with an identical parabolic mirror to a $1 \mathrm{~mm}$ thick $110 \mathrm{ZnTe}$ crystal for electro-optical detection with the weak $800 \mathrm{~nm}$ probe.

In Fig. 2, we present the waveforms of the THz field from the biased filaments as a function of the polarity and strength of the longitudinal electric field. All these $\mathrm{THz}$ waveforms are normalized to their maximum for a better view of the details. Therefore, a better signal-tonoise ratio corresponds to a stronger $\mathrm{THz}$ field when we compare these waveforms, since the noise level keeps constant for all the measurements. We note that the secondary $\mathrm{THz}$ peak around $\mathrm{t}=17 \mathrm{ps}$ correspond to the round-trip of the $\mathrm{THz}$ radiation inside the silicon filter due to the surface reflection. In the presence of a negative voltage (electric field in the direction of laser propagation), the $\mathrm{THz}$ field increases monotonically from $E_{e x}=0$ $\mathrm{kV} / \mathrm{cm}$ with a slight distortion up to $E_{e x}=9 \mathrm{kV} / \mathrm{cm}$ (Fig. 2(a)-(e)). In the opposite case (positive voltage), the evolution of the $\mathrm{THz}$ waveform is different. First of all, the $\mathrm{THz}$ signal decreases with increasing electric field up to $E_{e x} \sim 3 \mathrm{kV} / \mathrm{cm}$, which is evident from a much smaller SNR in Fig. 2 (h) comparing to Fig. 2 (a). With further increase of the field, the $\mathrm{THz}$ starts to increase again. At the same time, a complicated transformation of the $\mathrm{THz}$ waveform is noticeable. In the presence of relatively high field $\left(E_{e x} \geq 4.5 \mathrm{kV} / \mathrm{cm}\right)$, the $\mathrm{THz}$ waveforms finally exhibit an inverse polarity, when compared to positive voltage.

The peak-to-peak (PTP) amplitudes of the THz field are presented as a function of the external electric field in Fig. 3. In the presence of weak electric field, the PTP amplitude is not well defined due to the multiple peak structure of the $\mathrm{THz}$ field. Here, we just pick up the values of the maxima of the positive/negative peaks in relevant cases. We find that the $\mathrm{THz}$ amplitude depends on the field strength linearly, which is in agreement with our previous report of quadratic dependence of the THz intensity on electric field strength [17]. A minimum $\mathrm{THz}$ signal obtained around $E_{e x}=1.5 \mathrm{kV} / \mathrm{cm}$ is now evident. This means that the presence of a positive electric field largely suppresses the spontaneous electron motion inside a free plasma filament, indicating that its initial electron motion is pointed backward the laser propagation direction. We note that this is contradict to previous result which suggests that electrons inside a filament formed in $\mathrm{N}_{2}$ gas oscillate initially in the laser 
propagation direction [22]. However, the focusing geometry $(f=180 \mathrm{~mm})$ and the laser energy $(3 \mathrm{~mJ})$ are both different from that experiment $(f=1000 \mathrm{~mm}, 1 \mathrm{~mJ})$. As suggested in that work, the initial electron movement direction is determined by a delicate competition between the laser pressure and the resulting plasma wake force [22]. Therefore, we argue that the backwards electron motion the laser propagation direction can be due to much tight focusing, which may result in a stronger plasma wake force.

We note that in the presence of external field the $\mathrm{THz}$ field oscillation period increases gradually, by comparing Fig. 2 (a) and Fig. 2 (e). In Fig. 4 (a), we present the spectra of the $\mathrm{THz}$ radiation with and without the longitudinal field corresponding to the above two waveforms. A spectrum shift towards low frequency is noticeable, in the presence of an external field $E_{\text {ext }}=9 \mathrm{kV} / \mathrm{cm}$. This suggests that the frequency component of the plasma current induced by the external field is different from that induced by the laser pulse itself. Also, we would like to point out that the spectrum in Fig. 2 (j) is almost identical to that of Fig 2 (e), as expected from their similar temporal waveforms.

In the case of plasma filaments without external electric field, the longitudinal ponderomotive force of the rear side of the laser pulse accelerates the newly born electrons. It initializes an electron motion in the backwards direction, and creates an electrostatic field inside the filament in its wake. This charge separation in the longitudinal direction provokes an oscillating current. The Fourier spectrum of the current reads as $[17,23]$ :

$$
j_{z}^{w}(\omega)=\frac{e \omega_{p e}^{2} I_{0}}{2 m_{e} c^{2} \omega_{0}^{2}} \frac{\omega+2 i v_{e}}{\omega_{p e}^{2}-\omega^{2}+i v_{e} \omega} \frac{\sin \left(\omega \tau_{L} / 2\right)}{1-\left(\omega \tau_{L} / 2 \pi\right)^{2}} \exp \left(-\frac{i \omega \tau_{L}}{2}\right),
$$

where $I_{0}, \omega_{0}, v_{e}$ are the the laser intensity, the laser frequency and the electron collision frequency, respectively. The current spectrum has a maximum at the electron plasma frequency, $\omega_{p e}=\sqrt{e^{2} n_{e} / m_{e} \varepsilon_{0}}$, and its amplitude is proportional to the laser intensity.

The external longitudinal electric field is well below the discharge threshold and it does not affect the ionization process in air driven by the laser pulse. Nevertheless, the external field $E_{\text {ext }}$ can easily exceed the wake electric field $E_{w}$ created by the laser ponderomotive 
force. As a result, the electrons ionized by the laser field are now accelerated by the total longitudinal electric field, $E_{\text {ext }}+E_{w}$, originated from the laser ponderomotive force and the external field. The current spectrum due to the external electric field has been obtained as:

$$
j_{Z}^{e}(\omega)=\frac{\varepsilon_{0} \omega_{p e}^{2} E_{e x t}}{\omega^{2}-\omega_{p e}^{2}+i v_{e} \omega},
$$

which depends on the external field $E_{\text {ext }}$ linearly [17]. This explains naturally the experimentally observed linear dependence of the $\mathrm{THz}$ amplitude on the external field presented in Fig. 3. The absolute value of the current spectra given by expressions (1) and (2) are plotted in Fig. 4 (b). Good agreement is found with our experimental observation by adopting an electron density of $0.9 \times 10^{16} / \mathrm{cm}^{3}$ and a collision frequency of $v_{e}=0.35$ $\mathrm{THz}$, which are typical values for the plasma filaments formed in ambient air [24, 25]. Qualitatively speaking, the spectra in Eqs (1) and (2) are different because the laser ponderomotive force is of a short duration ( $\sim 40 \mathrm{fs})$ thus giving additional $\omega$ in the numerator in $\mathrm{Eq}$ (1). In opposite, the external field is static, its Fourier transformation gives $1 / \omega$ in the spectrum thus downshifting the spectrum.

In conclusion, we measured the $\mathrm{THz}$ waveform from femtosecond filaments which is longitudinally biased with an external electrical field. A complicated $\mathrm{THz}$ waveform distortion is observed in the presence of relatively weak electric field $\left(E_{e x}<4.5 \mathrm{kV} / \mathrm{cm}\right)$. A minimum $\mathrm{THz}$ signal is observed with electric field in the opposite direction of laser propagation with strength of $E_{e x}=1.5 \mathrm{kV} / \mathrm{cm}$, suggesting the pondermotive force induces electron motion inside a field free plasma filament is in the backward direction of laser propagation. We further compared the $\mathrm{THz}$ spectrum from the filaments with and without the external field. A spectrum shift towards a lower frequency is observed in the presence of the field, which is well explained by a theoretical model. 


\section{References:}

1. H. Hamster, A. Sullivan, S. Gordon, W. White, R. W. Falcone, Phys. Rev. Lett. 71, 2725 (1993)

2. D. J. Cook, R. M. Hochstrasser, Opt. Lett. 25, 1210 (2000).

3. K. Y. Kim, A. J. Talor, J. H. Glownia, G. Rordriguez, Nature Photon. 2, 605 (2008).

4. T. Löffler, F. Jacob, and H. G. Roskos, Appl. Phys. Lett. 77, 453 (2000).

5. J. Dai, N. Karpowicz, X. -C. Zhang, Phys. Rev. Lett.103, 023001(2009).

6. J. Liu, J. Dai, S. L. Chin, X. -C. Zhang, Nature Photonics 4, 627 (2010).

7. L. Berge, S. Skupin, C. Kohler, I. Babuchkin, and J. Herrmann, Phys. Rev. Lett. 110, 073901 (2013).

8. V. A. Andreeva, O. G. Kosareva, N. A. Panov, D. E. Shipilo, P. M. Solyankin, M. N. Esaulkov, P. González. de Alaiza Martinez, A. P. Shkurinov, V. A. Makarov, L. Bergé, and S. L. Chin, Phys. Rev. Lett. 116, 063902 (2016).

9. Z. Zhang, Y. Chen, S. Cui, F. He, M. Chen, Z. Zhang, J. Yu, L. Chen, Z. Sheng, and J. Zhang, Nat. Photonics 12, 554 (2018).

10. Y. J. Yoo, D. Jang, and K. Y. Kim, Opt. Express 27, 22663 (2019).

11. L. Zhang, W. Wang, T. Wu, R. Zhang, S. Zhang, C. Zhang, Y. Zhang, Z. Sheng, and X.-C. Zhang, Phys. Rev. Lett. 119, 235001 (2017).

12. C. D'Amico, A. Houard, M. Franco, B. Prade, A. Mysyrowicz, A. Couairon, and V. T. Tikhonchuk, Phys. Rev. Lett. 98, 235002 (2007).

13. T. J. Wang, J. F. Daigle, S. Yuan, F. Theberge, M. Chateauneuf, J. Dubois, G. Roy, H. Zeng, and S. L. Chin, Phys. Rev. A 83, 053801 (2011).

14. Aurélien Houard, Yi Liu, André Mysyrowicz, Bernadette Leriche, Appl. Phys. Lett. 90, 171501 (2007).

15. Mark D. Thomson, Markus Kre, Torsten Löffler, Hartmut G. Roskos, Laser Photon. Rev. 1, 349 (2007).

16. A. Houard, Y. Liu, B. Prade, V. Tikhonchuk, and A. Mysyrowicz, Phys. Rev. Lett. 100, 255006 (2008).

17. Y. Liu, A. Houard, B. Prade, A. Mysyrowicz, A. Diaw, V. Tikhonchuck, Appl. Phys. Lett. 93, 051108 (2008).

18. Y. Liu, A. Houard, B. Prade, S. Akturk, A. Mysyrowicz, and V. Tikhonchuk, Phys. Rev. Lett. 99, 135002 (2007).

19. P. G. Martinez, I. Babushkin, L. Bergé, S. Skupin, E. Cabrera-Granado, C. Kohler, U. Morgner, A. Husakou, and J. Herrmann, Phys. Rev. Lett. 114, 183901 (2015). 
20. V. Vaičaitis, O. Balachninaitė, U. Morgner, and I. Babushkin, J. Appl. Phys. 125, 173103 (2019).

21. C. Lu, C. Zhang, L. Zhang, X. Wang, and S. Zhang, Phys. Rev. A 96, 053402 (2017).

22. B. Zhou, A. Houard, Y. Liu, B. Prade, A. Mysyrowicz, A. Couairion, P. Mora, C. Smeenk, L. Arissian, P. Corkum, Phys. Rev. Lett. 106, 255002(2011).

23. C. D. Amico, A. Houard, S. Akturk, Y. Liu, J. Le. Bloas, M. Franco, B. Prade, A. Couairon, V. T. Tikhonchuk, and A. Mysyrowicz, New J. Phys. 10, 013015 (2008).

24. A. Couairon and A. Mysyrowicz, Phys. Rep. 441, 47 (2007).

25. S. L. Chin, S. A. Hosseini, W. Liu, Q. Luo, F. Théberge, N. Aközbek, A. Becker. V. P. Kandidov, O. G. Kosareva, and H. Schroder, Can. J. Phys. 83, 863 (2005). 


\section{Figure 1}

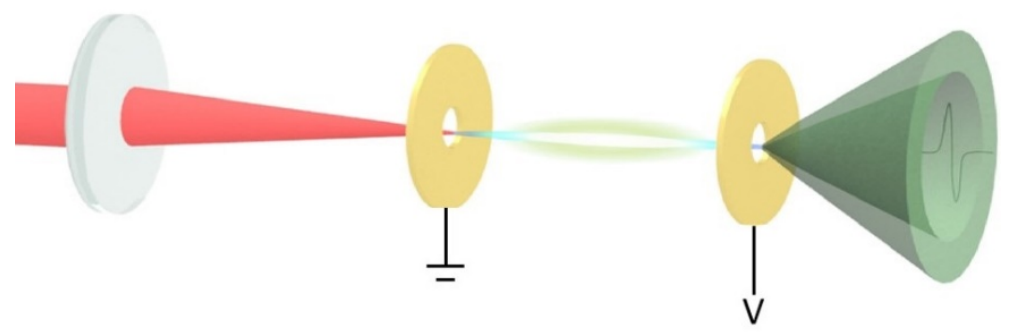

Fig. 1. Schematic experiment setup for the electric field biased filaments. The femtosecond laser pulse propagates from the left to the right. A pair of circular electrodes with central holes was mounted on the filament to apply a longitudinal electric field. 
Figure 2
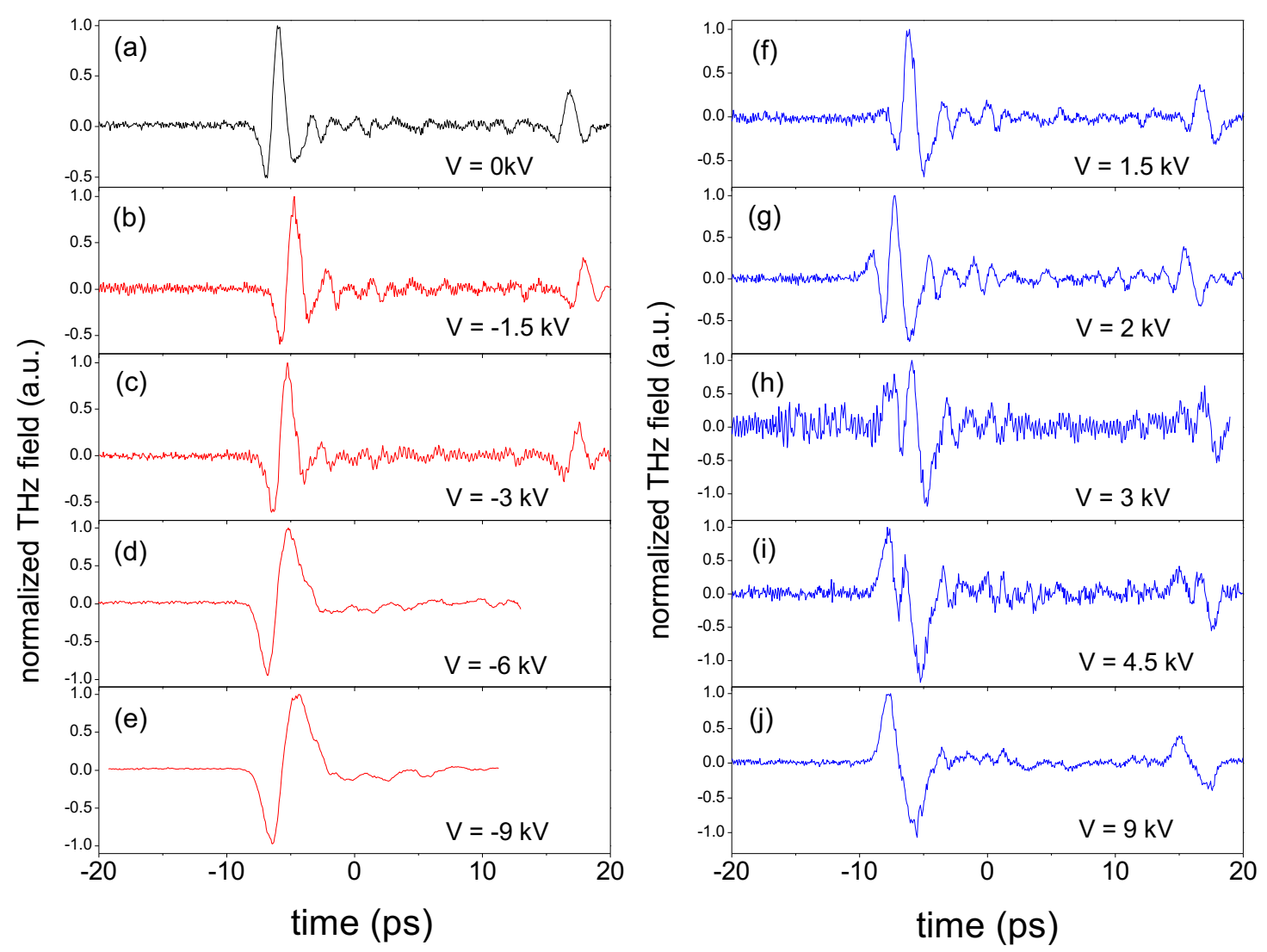

Figure 2. Waveforms of the THz field from the biased filaments. (a) no external field, (b)- (e) electric field in the direction of laser propagation, (f)-(j), with electric field in the opposite direction of laser propagation. The applied voltages on the $15 \mathrm{~mm}$ separated plate electrodes are indicated in each panel. 
Figure 3

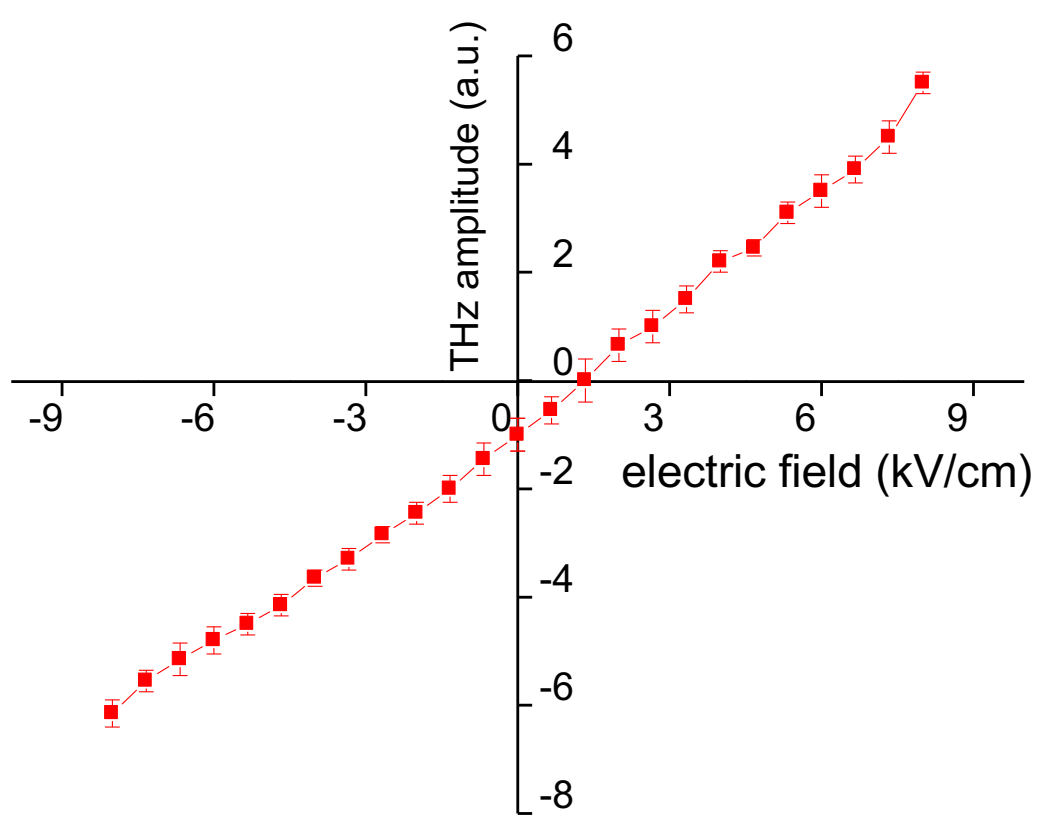

Figure 3. Peak-to-peak amplitude of the THz field as a function of the external electric field. The error bars denote the standard derivation of the measured signal. 
Figure 4
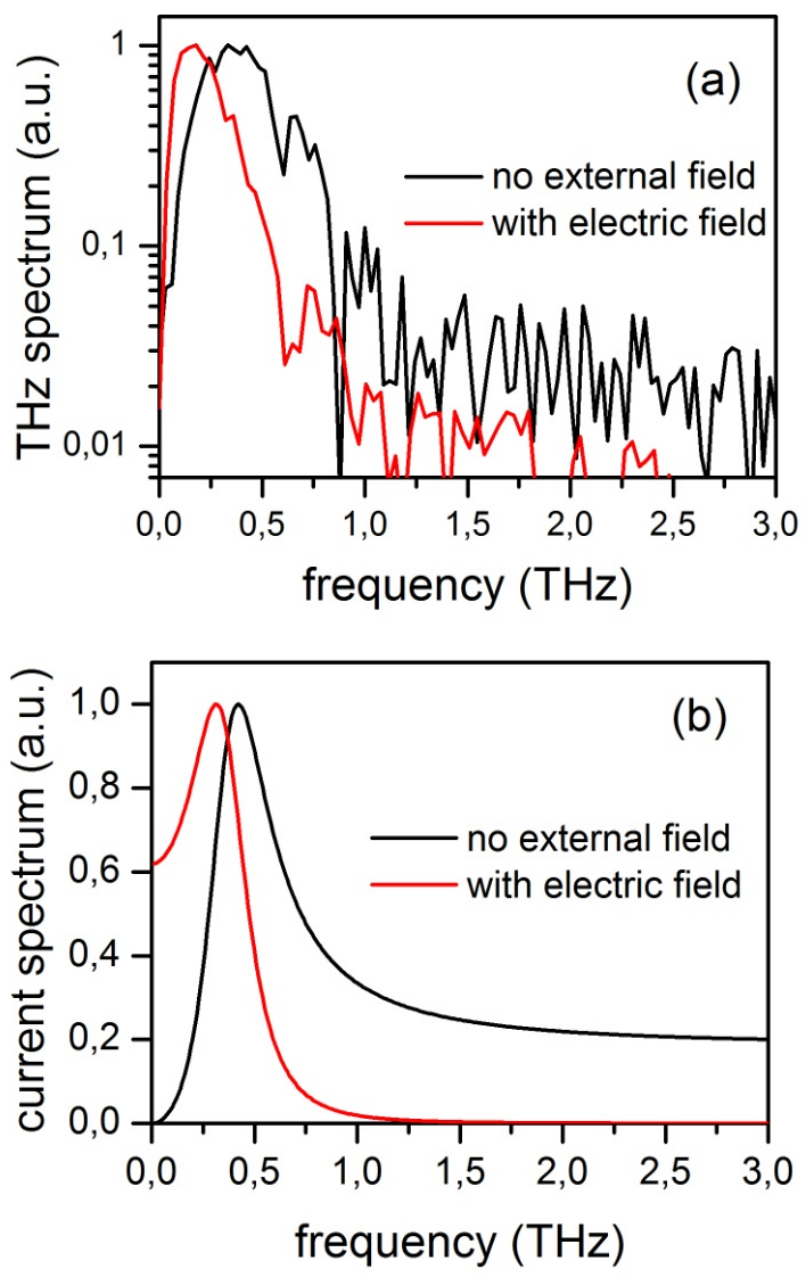

Figure 4. (a) $\mathrm{THz}$ spectrum in the presence of the electric field of $E_{e x}=-6 \mathrm{kV} / \mathrm{cm}$ (red thin line) and that without the field (black intensified line). (b) calculated plasma current spectrum with (red thin line) and without (black line) the bias electric field. 